\title{
Fuzzy grey predictor compensated time-varying variable structure controller for solar inverters
}

\author{
En-Chih Chang ${ }^{\mathrm{a}}$, Ziang $\mathrm{Xu}^{\mathrm{a}}$, Zhiyi $\mathrm{Su}^{\mathrm{b}}$ and Rong-Ching $\mathrm{Wu}^{\mathrm{a}, *}$ \\ ${ }^{a}$ Department of Electrical Engineering, I-Shou University, Dashu District, Kaohsiung City, Taiwan, R.O.C. \\ ${ }^{\mathrm{b}}$ School of Control Science and Engineering of Shandong University, Jinan, P.R.C.
}

\begin{abstract}
In this paper, a fuzzy grey predictor (GP) compensated time-varying variable structure controller (TVVSC) is developed and applied to solar inverters. TVVSC can shorten the reaching phase and ensure the sliding mode occurrence from an arbitrary initial state. However, while loading is a severe nonlinear condition, the TVVSC may suffer from chattering and steady-state error problems, thus deteriorating solar inverter performance. A GP is thus devoted to alleviate the chattering when the system uncertainty bounds are overestimated, and to reduce the steady-state error when the system uncertainty bounds are underestimated. However, the GP with a fixed forecasting value causes long rise time or large overshoot of the system response. Thus, fuzzy logic (FL) is applied to obtain flexible forecasting values to improve the system performance. With the proposed controller, the robustness of the solar inverter system can be enhanced, and a high-quality solar inverter sinusoidal output voltage with low voltage harmonics and fast dynamic response can be obtained, even under nonlinear loading. The theoretical analysis, design procedure, computer simulations, and digital signal processing (DSP)-based experimental implementation for solar inverters are presented to verify the efficacy of the proposed controller.
\end{abstract}

Keywords: Grey predictor (GP), time-varying variable structure controller (TVVSC), fuzzy logic (FL), solar inverter, voltage harmonics

\section{Introduction}

In recent years, solar inverters have gained increasing attention, and been broadly used in energy conversion systems [4]. In solar energy systems, the overall performance is dependent upon the static inverter-filter arrangement, which is used to convert a DC voltage into a sinusoidal $\mathrm{AC}$ output. The requirements for a highperformance solar inverter must supply a high-quality

\footnotetext{
${ }^{*}$ Corresponding author. Rong-Ching Wu, Department of Electrical Engineering, I-Shou University, No.1, Sec. 1, Syuecheng Rd., Dashu District, Kaohsiung City 84001, Taiwan, R.O.C. Tel.: +886 7 6577711//Ext: 6642; Fax: +886 7 6577205; E-mail: rcwu@isu.edu.tw.
}

AC output voltage with low total harmonic distortion (THD), zero steady-state error and a fast dynamic response; these can be obtained by employing feedback control techniques. The linear proportional Integral (PI) controller is typically used. However, the classic PI controlled system can not ensure fast and stable output voltage response [7,9]. Various control techniques have been proposed, such as wavelet transform technique, deadbeat control, and repetitive control, etc. However, these control techniques are difficult to implement and complex in computation [3, 10, 13]. For robust control systems, the variable structure control (VSC) can be adopted because the VSC is insensitive to system 
uncertainties, and provides a fast dynamic response. The controller of the solar inverters is also very popularly designed with VSC; however, the design of the classic VSC with fixed sliding surface is employed, and the sliding mode is attained when the system state reaches and maintains on the intersection of the sliding surface $[1,14]$. Thus, the trajectories are sensitive to uncertainties before the sliding mode occurs. To improve such problems, the concept of the TVVSC is introduced. The TVVSC is first chosen to pass arbitrary initial conditions and subsequently moved towards a predetermined sliding surface by means of rotating and/or shifting. By employing the time-varying sliding surface, the system sensitivity to uncertainties is reduced by shortening the reaching phase $[8,12]$. However, once a highly nonlinear loading is applied, the TVVSC controlled system is subject to chattering and steady-state error, thus resulting in serious voltage harmonics in the solar inverter output, even deteriorating the solar inverter performance. The grey predictor (GP) has been proposed by Deng, and many applications in a variety of fields have been developed. The grey model is built based on first-order differential equations, and utilizes mathematical approximation to transfer a continuous form into a discrete form. Such a transformation will confront some unconquerable problems, such as limited sampling frequency, sample/hold effects and discretization errors. Use of a difference equation replaces the differential equation to build a grey model that provides a reasonable and exact approach [5, 11]. This paper employs a mathematically simple and computationally efficient GP to alleviate the chattering and steady-state error when the system uncertainties are overestimated or underestimated. Though GP requires several output data to achieve a grey model and to forecast a future value without complex calculation, the GP uses a fixed forecasting value and leads to long rise time or large overshoot of the system response. Fuzzy set theory was introduced in 1965 and has received extensive applications. Fuzzy logic (FL) is thus used to tune the GP flexible forecasting value so as to maintain both a short rise time and a small overshoot of solar inverters system response $[2,6]$. By combining FL, GP, and TVVSC, the proposed controller will yield a closed-loop solar inverter with low THD, fast dynamic response, elimination of chatter, and steady-state error reduction under various types of loads. Simulation and experimental results are demonstrated to verify the performance of the proposed controller.

\section{Solar inverter control design}

A representative single-phase solar inverter is shown in Fig. 1, with a DC power supply $V_{d c}$, resistance load $R$, PWM full-bridge inverter and $L-C$ filter. The proposed controller is used to design a $1 \mathrm{KW} 60 \mathrm{~Hz}$ solar inverter.

The $L-C$ filter and $R$ are considered to be a plant system, and the dynamics with state variables are expressed as:

$$
\dot{x}_{p 2}=-a_{p 1} x_{p 1}-a_{p 2} x_{p 2}+b_{p} u_{p}+d
$$

where $x_{m}=\left[\begin{array}{ll}x_{p 1} & x_{p 2}\end{array}\right]=\left[v_{c} \dot{v}_{c}\right], a_{p 1}=\frac{1}{L C}, a_{p 2}=$ $\frac{1}{R C}, b_{p}=\frac{K_{P W M}}{L C}, K_{P W M}$ is the proportional gain of the inverter, $u_{p}$ is the plant input, and $d$ is the uncertainty.

The model system is given by the sinusoidal function with $60 \mathrm{~Hz}$.

$$
\dot{x}_{m 2}=-a_{m 1} x_{m 1}-a_{m 2} x_{m 2}+b_{m} u_{m}
$$

where, $x_{m}=\left[\begin{array}{ll}x_{m 1} & x_{m 2}\end{array}\right]=\left[\begin{array}{ll}v_{r e f} & \dot{v}_{r e f}\end{array}\right], \quad a_{m 1}=\omega_{0}^{2}=$ $(2 \pi \cdot 60)^{2}, a_{m 2}=0, b_{m}=1$, and $u_{m}$ is the reference input.

By defining $e_{1}=x_{m 1}-x_{p 1}$, subtracting Equation (1) from Equation (2), the error differential equation yields:

$$
\begin{aligned}
\dot{e}_{1}= & e_{2}=\dot{x}_{m 1}-\dot{x}_{p 1}=x_{m 2}-x_{p 2} \\
\dot{e}_{2}= & -a_{m 1} e_{1}-a_{m 2} e_{2}+\left(a_{p 1}-a_{m 1}\right) x_{p 1} \\
& +\left(a_{p 2}-a_{m 2}\right) x_{p 2}+b_{m} u_{m}-b_{p} u_{p}-d
\end{aligned}
$$

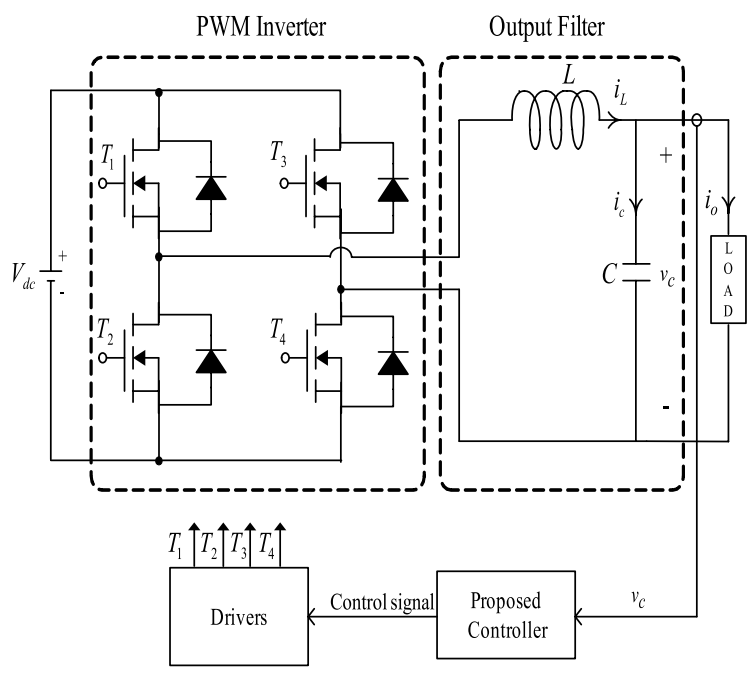

Fig. 1. Block diagram of solar inverter. 
Then, the time-varying sliding surface is selected as:

$$
\sigma=c_{1}(t) e_{1}+e_{2}-\alpha(t)
$$

where $c_{1}(t)=A t+B ; \alpha(t)=C t+D ;$ and $A, B, C, D$ are constants.

Taking the derivative of $\sigma$ yields:

$$
\begin{aligned}
\dot{\sigma}= & A e_{1}+c_{1}(t) e_{2}-a_{m 1} e_{1}-a_{m 2} e_{2}+\left(a_{p 1}-a_{m 1}\right) x_{p 1} \\
& +\left(a_{p 2}-a_{m 2}\right) x_{p 2}+b_{m} u_{m}-b_{p} u_{p}-d-C \quad(6)
\end{aligned}
$$

Define $a_{p_{i}}=a_{p i}^{0}+\Delta a_{p i}$ and $b_{p}=b_{p}^{0}+\Delta b_{p}$, and the control function can be expressed as:

$$
u_{p}=u_{e}+u_{s}
$$

The equivalent control $u_{e}$, which can determine the dynamic of the system on the sliding surface is derived as:

$$
\begin{aligned}
& A e_{1}+c_{1}(t) e_{2}-a_{m 1} e_{1}-a_{m 2} e_{2}+\left(a_{p 1}-a_{m 1}\right) x_{p 1} \\
& +\left(a_{p 2}-a_{m 2}\right) x_{p 2}+b_{m} u_{m}-b_{p} u_{p}=0
\end{aligned}
$$

Substituting $u_{p}=u_{e}$ into Equation (8) yields:

$$
\begin{aligned}
u_{e}= & {\left[A e_{1}+c_{1}(t) e_{2}-a_{m 1} e_{1}-a_{m 2} e_{2}\right.} \\
& +\left(a_{p 1}^{0}-a_{m 1}\right) x_{p 1} \\
& \left.+\left(a_{p 2}^{0}-a_{m 2}\right) x_{p 2}+b_{m} u_{m}-C\right] / b_{p}^{0}
\end{aligned}
$$

When in sliding action:

$$
e_{2}=-c_{1}(t) e_{1}-\alpha(t)
$$

Substituting Equation (10) into Equation (9) yields:

$$
\begin{aligned}
& {\left[A e_{1}+c_{1}^{2}(t) e_{1}+\alpha(t) c_{1}(t)-a_{m 1} e_{1}\right.} \\
u_{e}= & +c_{1}(t) a_{m 2} e_{1}+\alpha(t) a_{m 2}+\left(a_{p 1}^{0}-a_{m 1}\right) x_{p 1} \\
+ & \left.+\left(a_{p 2}^{0}-a_{m 2}\right) x_{p 2}+b_{m} u_{m}-C\right]
\end{aligned}
$$

The system performance can be ensured despite the existence of the uncertain system dynamics. The sliding control $u_{s}$ is designed as follows:

$$
u_{s}=K_{1} e_{1}+K_{2} e_{2}+K_{n}
$$

Inserting Equation (12) in $\sigma \dot{\sigma}<0$, sufficient stability conditions $K_{1}, K_{2}$ and $K_{n}$ will be obtained.

Notice that if a highly nonlinear loading is connected to solar inverter output, the chattering and steady-state error will occur. Thus, we employ the $\operatorname{GP}$ with $\operatorname{GM}(2,1)$ model to eliminate the chattering and steady-state error. The GP modeling steps are described below.
Step 1. Gather the original sample data sequence

$$
x^{(0)}=\left\{x^{(0)}(k), k=1,2, \ldots, n\right\}
$$

Step 2. Mapping generating operation (MGO).

The data sequence of the system may be positive or negative. The MGO maps the negative data to the relative positive data.

$$
x_{\text {new }}^{(0)}=\left\{x^{(0)}(k)+\text { Bias, } k=1,2, \ldots, n\right\}
$$

where bias is constant.

Step 3. Take accumulated generating operation (AGO) for $x_{n e w}^{(0)}$

$$
x_{\text {new }}^{(1)}(k)=\sum_{i=1}^{k} x_{\text {new }}^{(0)}(i), k=1,2, \ldots, n
$$

Step 4. Build the $\operatorname{GM}(2,1)$ model.

The linear difference equation grey prediction model is established as:

$$
x_{\text {new }}^{(1)}(k+2)+M x_{\text {new }}^{(1)}(k+1)+N x_{\text {new }}^{(1)}(k)=0
$$

where $M$ and $N$ are the coefficients of the difference equation grey prediction model that must estimate their values.

The, Equation (16) is rewritten as the following matrix:

$$
\left[-x_{\text {new }}^{(1)}(k+1)-x_{\text {new }}^{(1)}(k)\right]\left[\begin{array}{l}
M \\
N
\end{array}\right]=\left[x_{\text {new }}^{(1)}(k+2)\right]
$$

Let $k=1,2, \ldots, n-2$ and suppose $Y=\left[\begin{array}{c}x_{\text {new }}^{(1)}(3) \\ x_{\text {new }}^{(1)}(4) \\ \vdots \\ x_{\text {new }}^{(1)}(n)\end{array}\right]$,

$B=\left[\begin{array}{cc}-x_{\text {new }}^{(1)}(2) & -x_{\text {new }}^{(1)}(1) \\ -x_{\text {new }}^{(1)}(3) & -x_{\text {new }}^{(1)}(2) \\ \vdots & \vdots \\ -x_{\text {new }}^{(1)}(n-1) & -x_{\text {new }}^{(1)}(n-2)\end{array}\right]$,

and $\Theta=\left[\begin{array}{c}M \\ N\end{array}\right]$, then the estimated parameters $M$ and $N$ can be solved by the least square estimation method as follows:

$$
\Theta=[M, N]^{T}=\left(B^{T} B\right)^{-1} B^{T} Y
$$


Let $x_{n e w}^{(1)}(k)=\xi^{k}, x_{n e w}^{(1)}(k+1)=\xi^{k+1}$, and $x_{n e w}^{(1)}(k+$ $2)=\xi^{k+2}$; the following equation is obtained:

$\xi^{k+2}+M \xi^{k+1}+N \xi^{k}=\xi^{k}\left(\xi^{2}+M \xi+N\right)=0$

The roots to satisfy Equation (19) are given as:

$$
\left\{\begin{array}{l}
\xi_{1}=\frac{-M+\sqrt{M^{2}-4 N}}{2} \\
\xi_{2}=\frac{-M-\sqrt{M^{2}-4 N}}{2}
\end{array}\right.
$$

If $\xi_{1} \neq \xi_{2}$, the solution of the prediction model of the second-order difference equation can be expressed as:

$$
\hat{x}_{n e w}^{(1)}(k)=C_{1} \xi_{1}^{k}+C_{2} \xi_{2}^{k}
$$

where " $\wedge$ " represents the predicted value, and $C_{1}$ and $C_{2}$ are constants.

If $\xi_{1}=\xi_{2}$, the solution of the prediction model of the second-order difference equation can be expressed as:

$$
\hat{x}_{\text {new }}^{(1)}(k)=C_{1} \xi_{1}^{k}+C_{2} k \xi_{1}^{k}
$$

If $\xi_{1}$ and $\xi_{2}$ are complex conjugate, the solution of the prediction model of the second-order difference equation can be expressed as:

$$
\hat{x}_{n e w}^{(1)}(k)=C_{1} v^{k} \sin \phi k+C_{2} v^{k} \cos \phi k
$$

Step 5. Take inverse accumulated generating operation (IAGO).

$$
\hat{x}_{\text {new }}^{(0)}(k)=\hat{x}_{\text {new }}^{(1)}(k)-\hat{x}_{\text {new }}^{(1)}(k-1)
$$

Step 6. Inverse mapping generating operation (IMGO). Applying IMGO to $\hat{x}_{n e w}^{(0)}$, the predicted value of the original data sequence $\hat{x}^{(0)}$ is obtained as:

$$
\hat{x}^{(0)}(k+1)=\hat{x}_{n e w}^{(1)}(k+1)-\hat{x}_{n e w}^{(1)}(k)-\text { Bias }
$$

Then, the control law of Equation (7) is restated as:

$$
u_{p}(k)=u_{e}(k)+u_{s}(k)+u_{g}(k)
$$

where the grey prediction control $u_{g}$ assists in alleviating chatter and steady-state error.

$$
u_{g}(k)=\left\{\begin{array}{cc}
0 & ,|\hat{\sigma}(k)| \leq \varepsilon \\
K \hat{\sigma}(k) \operatorname{sgn}(\sigma(k) \hat{\sigma}(k)), & |\hat{\sigma}(k)| \geq \varepsilon
\end{array}\right.
$$

where $K$ is constant, $\hat{\sigma}(k)$ represents the predicted value of $\sigma(k)$, and $\varepsilon$ indicates the system boundary. Note that the control law in Equation (7) implies the signum function $(\operatorname{sgn}(\cdot))$ and requires infinite switching frequency in the theory, to maintain the system states on the sliding surface. However, an infinite switching frequency cannot be realized when the control law is implemented on a DSP. Thus, using a saturation function $\operatorname{sat}(\cdot)$ instead of $\operatorname{sgn}(\cdot)$ provides:

$$
\operatorname{sat}(\hat{\sigma}(k), \varepsilon)=\left\{\begin{array}{cc}
\operatorname{sgn}(\sigma(k)) & \text { if }|\hat{\sigma}(k)|>\varepsilon \\
\hat{\sigma}(k) / \varepsilon & \text { if }|\hat{\sigma}(k)| \leq \varepsilon
\end{array}\right.
$$

It is worth noting that the above-mentioned GP uses a fixed forecasting value, and may cause long rise time or large overshoot of the system response. To solve such a problem, FL is introduced to finely tune the GP forecasting value, thus enhancing the system robustness. The operation of fuzzy tuning GP forecasting value is illustrated as follows. Let the predicted error and the change of the predicted error at the $k$ th-step ahead be $\hat{e}_{1}(k+1)$ and $\Delta \hat{e}_{1}(k+1)$, respectively. Define $\hat{e}_{1}$ and $\Delta \hat{e}_{1}$ as the input of the fuzzy logic, where the $\hat{e}_{1}$ and $\Delta \hat{e}_{1}$ are the fuzzy variables of the GP value $\hat{e}_{1}(k+1)$ and $\Delta \hat{e}_{1}(k+1)$, respectively. The output of the fuzzy logic $\left(U_{F L}\right)$ is the fuzzy variable indicating the forecasting value. The universes of discourse of $\hat{e}_{1}, \Delta \hat{e}_{1}$ and $U_{F L}$ are partitioned into the following fuzzy sets: NB (Negative Big); NM (Negative Medium); NS (Negative Small); Z (Zero); PB (Positive Big); PM (Positive Medium); PS (Positive Small); VS (Very Small); SM (Small); MD (Medium); LG (Large); VL (Very Large); HU (Huge); and VH (Very Huge); Thus, the FL of a two-input single-output fuzzy model with 49 rules can be obtained as follows:

$R^{1}$ : If $\hat{e}_{1}$ is NB and $\Delta \hat{e}_{1}$ is NB then $U_{F L}$ is $\mathrm{VH}$.

$R^{2}$ : If $\hat{e}_{1}$ is NM and $\Delta \hat{e}_{1}$ is NB then $U_{F L}$ is HU.

$R^{49}$ : If $\hat{e}_{1}$ is $\mathrm{PB}$ and $\Delta \hat{e}_{1}$ is $\mathrm{PB}$ then $U_{F L}$ is $\mathrm{VH}$.

\section{Simulation and experimental results}

The system parameters of the solar inverter are given as follows: DC-bus voltage, $V_{d c}=200 \mathrm{~V}$; switching frequency, $f_{s}=15 \mathrm{kHz}$; output voltage and frequency, $v_{c}=110 \mathrm{~V}_{\mathrm{rms}}, f=60 \mathrm{~Hz}$; rated resistive load, $R=12 \Omega$; filter inductor, $L=1.5 \mathrm{mH}$; filter capacitor, $C=15 \mu \mathrm{F}$. Figure 2(a) depicts the simulated transient response with the load changing from no load to full load at $t=0.0208 \mathrm{~s}$ for the proposed controller. The transient response of the proposed controller is very fast, taking only a few sampling intervals to reach steady-state. On the contrary, the simulated waveforms shown in Fig. 2(b) with classic VSC indicates a significant voltage sag. A simulated performance testing, shown in Fig. 2(c) is achieved by applying a nonlinear load consisting of a diode bridge, a capacitor $(100 \mu \mathrm{F})$ and a 


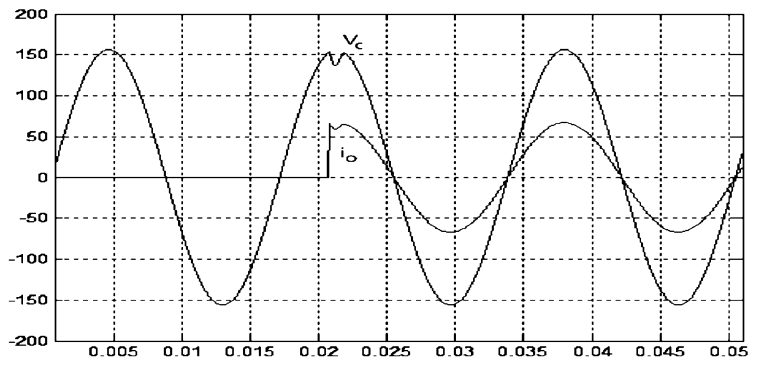

(a) $(50 \mathrm{~V} / \mathrm{div} ; 20 \mathrm{~A} / \mathrm{div})$.

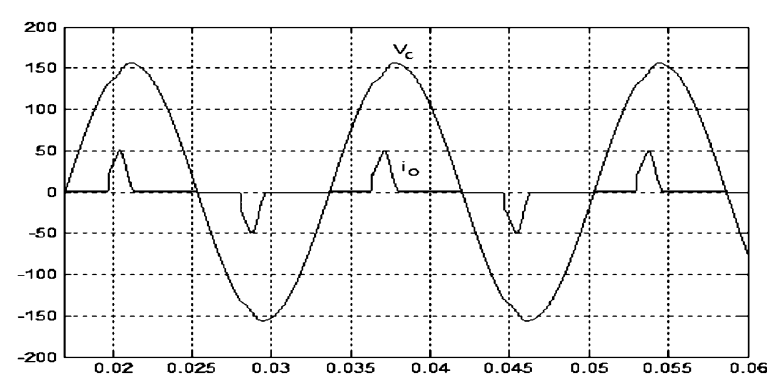

(c) $(50 \mathrm{~V} / \mathrm{div} ; 15 \mathrm{~A} / \mathrm{div})$.

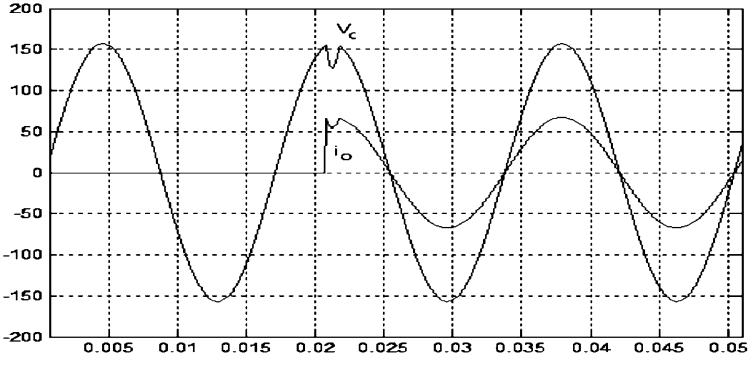

(b) $(50 \mathrm{~V} / \mathrm{div} ; 20 \mathrm{~A} / \mathrm{div})$.

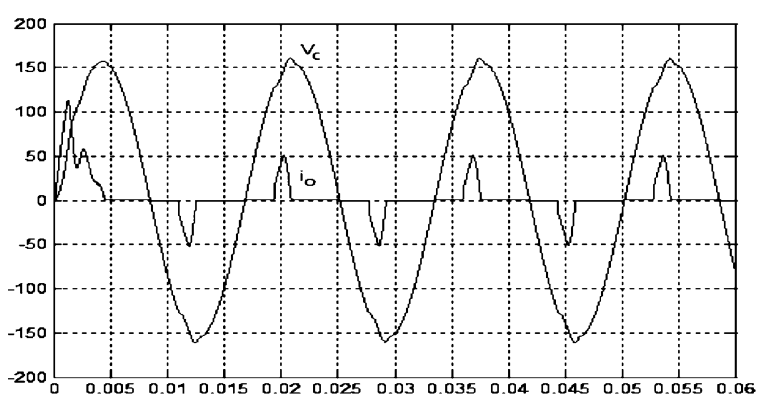

(d) $(50 \mathrm{~V} / \mathrm{div} ; 15 \mathrm{~A} / \mathrm{div})$.

Fig. 2. Depicts the simulation results of output voltage and load current under (a) step change in load with the proposed controller; (b) step change in load with classic VSC; (c) rectifier load with proposed controller; (d) rectifier load with classic VSC.

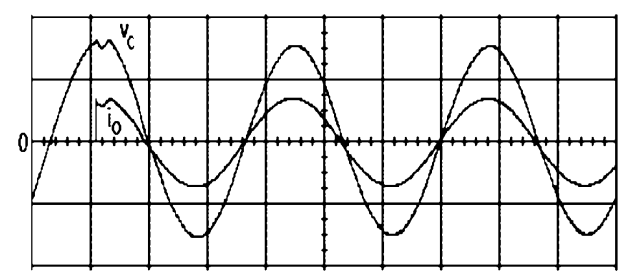

(a) $(100 \mathrm{~V} / \mathrm{div} ; 20 \mathrm{~A} / \mathrm{div} ; 5 \mathrm{~ms} / \mathrm{div})$.

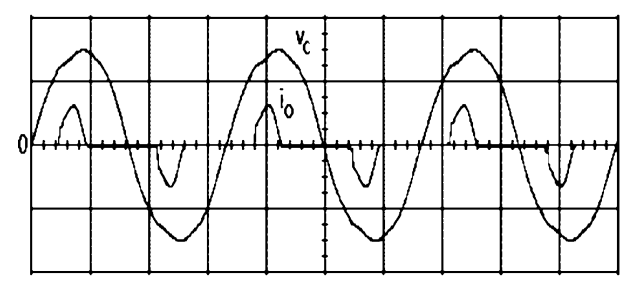

(c) $(100 \mathrm{~V} / \mathrm{div} ; 20 \mathrm{~A} / \mathrm{div} ; 5 \mathrm{~ms} / \mathrm{div})$.

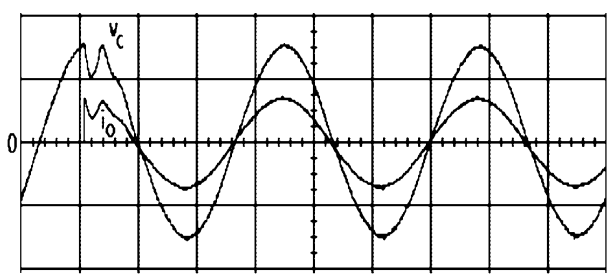

(b) $(100 \mathrm{~V} / \mathrm{div} ; 20 \mathrm{~A} / \mathrm{div} ; 5 \mathrm{~ms} / \mathrm{div})$.

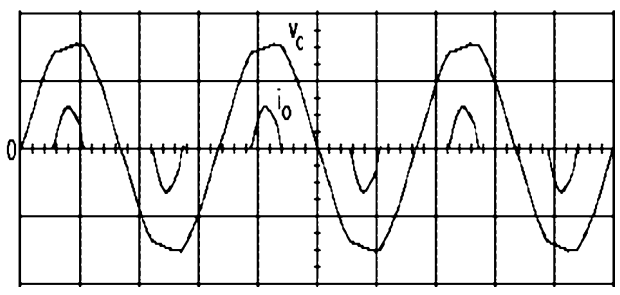

(d) $(100 \mathrm{~V} / \mathrm{div} ; 20 \mathrm{~A} / \mathrm{div} ; 5 \mathrm{~ms} / \mathrm{div})$.

Fig. 3. Experimental waveforms under (a) step change in load with the proposed controller; (b) step change in load with classic VSC; (c) rectifier load with proposed controller; (d) rectifier load with classic VSC.

resistive load $(60 \Omega)$. The simulated $\%$ THD computed in the output voltage is $1.13 \%$; The AC voltage is still satisfactory, and good behavior of the inverter is also obtained in this critical condition. However, under the same rectifier loading obtained using the classic VSC, shown in Fig. 2(d), exhibits a high simulated \%THD output voltage (\% THD of output voltage is equal to $8.01 \%$ ). Figure 3(a) displays the experimental output 
voltage and the load current with the proposed controller under a load step change from open circuit to $R=12 \Omega$. A rapid recovery of the steady-state response can be obtained. However, the experimental waveform with the classic VSC, shown in Fig. 3(b), demonstrates poor voltage compensation, especially at the firing angle. Additionally, the majority of sensitive loads are rectifier loads. When the diodes are conducting, the inverter is exposed to a large filter capacitor, and when the diodes are not conducting, the inverter is practically in no load condition. Therefore, the controller must correctly regulate output voltage with minimum distortion. Figure 3(c) shows the experimental output voltage and the load current with the proposed controller when the inverter is loaded with a full-wave rectifier followed by a $100 \mu \mathrm{F}$ capacitor in parallel with a $60 \Omega$ resistor; the experimental \% THD is close to $1.17 \%$, which indicates good inverter performance. On the contrary, Fig. 3(d) with the classic VSC under the same test condition exhibits a high experimental voltage \% THD of $7.42 \%$.

\section{Conclusions}

A fuzzy GP-compensated TVVSC has been proposed to improve the tracking behaviors of solar inverters. The TVVSC can shorten the reaching phase, thus enhancing the robustness of the system. However, with the application of a highly nonlinear loading, chattering and steady-state error occur. For high tracking accuracy, the GP is used to eliminate the chattering and steady-state error, which are produced by TVVSC. Additionally, FL is added to GP to tune the forecasting value, thus enhancing the system robustness and speed. Simulation and experimental results indicate that low THD, fast transient response, the elimination of the chattering, and the reduction of the steady-state error are obtained by the proposed controlled solar inverter under both steady-state loading and transient loading.

\section{Acknowledgments}

This work was supported by the Ministry of Science and Technology of Taiwan, R.O.C., under contract number MOST104-2221-E-214-011.

\section{References}

[1] A. Abrishamifar, A.A. Ahmad and M. Mohamadian, Fixed switching frequency sliding mode control for single-phase unipolar inverters, IEEE Trans on Power Electronics 27(5) (2012), 2507-2514.

[2] A. Syropoulos Theory of Fuzzy Computation Springer-Verlag, New York, 2014.

[3] D. Chen, J.M. Zhang and Z.M. Qian, An improved repetitive control scheme for grid-connected inverter with frequencyadaptive capability, IEEE Trans. on Industrial Electronics 60(2) (2013), 814-823.

[4] F.L. Luo and H. Ye Power electronics: Advanced conversion technologies, CRC Press 2010.

[5] G.D. Li, S. Masuda, D. Yamaguchi, M. Nagai and C.H. Wang, An improved grey dynamic $\operatorname{GM}(2,1)$ model, International Journal of Computer Mathematics 87(7) (2010), $1617-1629$.

[6] H. Abu-Rub, A. Iqbal, S. MoinAhmed, F.Z. Peng, Y. Li and B.M. Ge, Quasi-Z-source inverter-based photovoltaic generation system with maximum power tracking control using ANFIS, IEEE Trans on Sustainable Energy 4(1) (2013), $11-20$.

[7] I. Rasoanarivo, S. Brechet, A. Battiston and B. NahidMobarakeh, Behavioral analysis of a boost converter with high performance source filter and a fractional-order PID controller, Proc IEEE Int Conf Industry Applications Society Annual Meeting (2012), 1-6.

[8] J. Geng, Y.Z. Sheng and X.D. Liu, Second-order time-varying sliding mode control for reentry vehicle, International Journal of Intelligent Computing and Cybernetics 6(3) (2013), 272-295.

[9] R.S. Rebeiro and M.N. Uddin, Performance analysis of an FLC-based online adaptation of both hysteresis and PI controllers for IPMSM drive, IEEE Trans on Industry Applications 48(1) (2012), 12-19.

[10] S.A. Saleh and M.A. Rahman, Experimental performances of the single-phase wavelet-modulated inverter, IEEE Trans on Power Electronics 26(9) (2011), 2650-2661.

[11] S. Liu and Y. Lin Advances in Grey Systems Research, Springer-Verlag, Heidelberg, Berlin, 2010.

[12] S. Tokat, I. Eksin and M. Guzelkaya, Linear time-varying sliding surface design based on co-ordinate transformation for high-order systems, Transactions of the Institute of Measurement and Control 31(1) (2009), 51-70.

[13] X.G. Zhang, W.J. Zhang, J.M. Chen and D.G. Xu, Deadbeat control strategy of circulating currents in parallel connection system of three-phase PWM converter, IEEE Trans on Energy Conversion 29(2) (2014), 406-417.

[14] X. Hao, X. Yang, T. Liu, L. Huang and W.J. Chen, A slidingmode controller with multiresonant sliding surface for singlephase grid-connected VSI with an LCL filter, IEEE Trans on Power Electronics 28(5) (2013), 2259-2268. 